Recepción: 15 / 04 / 2017

Ciencias Médicas

Aceptación: 01 / 05 / 2017

Revisión de Literatura

Publicación: 15 / 05 / 2017

\title{
Técnicas de impresión alternativas en pacientes que presentan Síndrome de Combinación o Kelly
}

\section{Alternative Printing Techniques in Patients Who Have Combination Syndrome or Kelly}

Técnicas de impressão alternativos em pacientes com síndrome ou Kelly Combinação

\author{
Danny E. Romero-Luzuriaga ${ }^{\mathrm{I}}$ \\ dannyeduardo.romero@ug.edu.ec \\ Juan J. Valarezo-Torres II \\ juan.valarezot@ug.edu.ec \\ Katuska P. Velasco-Cornejo ${ }^{\text {III }}$ \\ katuska.velascoc@ug.edu.ec \\ Gloria M. Concha-Urgiles ${ }^{\text {IV }}$ \\ gloria.conchau@ug.edu.ec
}

Correspondencia: dannyeduardo.romero@ug.edu.ec

\footnotetext{
Especialista en Rehabilitación Oral; Odontólogo; Universidad de Guayaquil, Ecuador.

II. Magister en Educación Superior; Especialista en Proyectos de Desarrollo Educativos y Sociales; Doctor en Odontología; Universidad de Guayaquil, Ecuador.

III. Especialista en Odontopediatria; Diploma Superior en Diseño Curricular por Competencias; Doctora en Odontología; Universidad de Guayaquil, Ecuador.

Iv. Especialista en Rehabilitación Oral Integral; Odontóloga; Universidad de Guayaquil, Ecuador.
} 


\section{Resumen}

Elliswoth Kelly en 1972 fue quien definió el término de "Síndrome de Combinación" refiriéndose a pacientes que presentaban edentulismo total superior con una considerable perdida ósea maxilar anterior caracterizados por antagonizar con dientes anteroinferiores con prótesis parcial inferior a extensión distal, esta se produce porque el paciente al no tener superficie oclusal posterior utiliza el sector anterior en exceso lo que a su vez causa presión y reabsorción en el sector anterosuperior y radiográficamente lo podemos observar como una v invertida. Un alto índice de Profesionales de la cavidad bucal desconocen cómo tratar a un paciente que presenta el Síndrome de Kelly, Esta situación clínica trae como consecuencia molestia al paciente al momento de la toma de impresión ya que ellos presentan tejidos móviles e hipertrofia de la tuberosidad del maxilar lo cual nos impide tomar la impresión con la técnica convencional por lo tanto no vamos a obtener un buen registro de las estructuras bucales del paciente y por consiguiente no tendremos una buena adaptación de la prótesis. El objetivo de esta investigación es dar a conocer las diferentes alternativas de técnicas de impresión que hay para la buena adaptación de prótesis totales para pacientes que padecen esta patología y que ellos sean capaces de elegir la que mejor les favorezca ya sea por el tiempo o dinero. El método de investigación que se uso fue documental, descriptiva, correlacional y explicativa. Como conclusión decimos que la cubeta indicada para los pacientes que padecen esta patología es la cubeta individualizada ya que va a registrar los detalles anatómicos de cada paciente y así no tendremos problemas si la cubeta es grande o pequeña para nuestro paciente.

Palabras clave: Reabsorción; síndrome; edentulismo; funcional. 


\begin{abstract}
Elliswoth Kelly in 1972 defined the term "Combination Syndrome" referring to patients who had upper total edentulism with considerable anterior maxillary bone loss characterized by antagonizing with anteroinferior teeth with partial inferior prosthesis to distal extension, this occurs because the patient having no posterior occlusal surface using the anterior excess which in turn causes pressure and resorption in the anterior sector and we can observe radiographically as an inverted v. A high rate of oral cavity Professional know how to treat a patient with the syndrome Kelly, This clinical situation brings as a result the patient discomfort when taking the impression because they have mobile tissue and hypertrophy of the tuberosity jaw which prevents us from taking the impression to the conventional technique so we will not get a good record of the oral structures of the patient and therefore does not have a good adaptation of the prosthesis. The objective of this research is to present the different alternatives printing techniques there for good adaptation of dentures for patients suffering from this disease and that they are able to choose which best favor them either time or money. The research method used was documentary, descriptive, correlational and explanatory. In conclusion we say that the bucket indicated for patients suffering from this disease is individualized bucket as it will record the anatomical details of each patient and we will not have problems if the bucket is large or small for our patient.
\end{abstract}

Key words: Syndrome; denture; edentulous; pathology. 


\section{Resumo}

Elliswoth Kelly, em 1972, definiu o termo "Síndrome de Combinação", referindo-se a pacientes com edentulismo total superior com perda óssea maxilar anterior considerável, caracterizado por antagonizar com dentes anteroinferiores com prótese parcial inferior à extensão distal, o que ocorre porque o paciente não possui superfície oclusal posterior $\mathrm{O}$ excesso anterior que, por sua vez, provoca pressão e reabsorção no setor anterior e podemos observar radiograficamente como um invertido v. Uma alta taxa de cavidade oral Profissional sabe como tratar um paciente com a síndrome Kelly, Esta situação clínica traz como resultado a Desconforto do paciente ao tomar a impressão porque têm tecido móvel e hipertrofia da mandíbula da tuberosidade que nos impede de levar a impressão para a técnica convencional para que não consigamos um bom registro das estruturas orais do paciente e, portanto, não tem um bom Adaptação da prótese. O objetivo desta pesquisa é apresentar as diferentes alternativas técnicas de impressão lá para uma boa adaptação de próteses dentárias para pacientes que sofrem desta doença e que eles são capazes de escolher qual deles / delas melhor favorecem eles tempo ou dinheiro. O método de pesquisa utilizado foi documental, descritivo, correlacional e explicativo. Em conclusão, dizemos que o balde indicado para os pacientes que sofrem desta doença é individualizado balde como ele vai gravar os detalhes anatômicos de cada paciente e não teremos problemas se o balde é grande ou pequeno para o nosso paciente

Palavras chave: Síndrome; dentadura; desdentado; patologia. 


\section{Introducción.}

El Síndrome de Kelly se presenta cuando se usa una prótesis total versus una prótesis parcial inferior con dientes remanentes superiores y así los pacientes tienen un conjunto de signos clínicos, esto se produce porque al no tener superficie oclusal posterior utiliza el sector anterior en exceso lo que a su vez causa presión y reabsorción en el sector anterosuperior. Al no saber cómo actuar ante un paciente que presente síndrome de Kelly, he decidido realizar el siguiente trabajo de titulación para concientizar a los profesionales de la salud bucal en la manera correcta de la toma de impresión para obtener una buena adaptación en pacientes que padecen esta patología ${ }^{1,2}$.

La pérdida de hueso en la región anterior del maxilar edéntulo cuando antagoniza con dientes anteriores remanentes mandibulares es una de las características más importantes observadas en el síndrome de combinación ${ }^{3,4}$.

Aun cuando en el campo de la odontología se han producido muchos avances, este problema aun es una realidad a la que deben enfrentarse tanto el odontólogo como el paciente. Por ello es importante conocer el manejo y la prevención de los cambios degenerativos secundarios presentes en esta condición ${ }^{5,6}$.

Es esencial identificar los signos que caracterizan el síndrome de combinación e implementar las medidas correctivas necesarias para no inducir o empeorar la condición bucal del paciente, ya de por si desfavorable ${ }^{3}$.

El presente trabajo tiene como objetivo es del presente trabajo es determinar la alternativa de técnica de impresión superior para un paciente que padece Síndrome de Kelly, para mejorar la adaptación de la prótesis para obtener una elaboración correcta, funcional y estéticas de las prótesis 
Danny E. Romero-Luzuriaga; Juan J. Valarezo-Torres; Katuska P. Velasco-Cornejo; Gloria M. Concha-Urgiles

totales superiores de pacientes que presentan el Síndrome de Kelly, estableciendo el tiempo de adaptación de la prótesis dependiendo de la técnica de impresión por lo tanto elegiremos el material de impresión más adecuado según la necesidad de cada paciente ${ }^{7}$.

Los tipos de investigación que se usaron en este trabajo de titulación fueron documental, recopilando la información de textos, revistas o artículos, también fue descriptiva ya que analizamos y describimos el tema en cuestión y fue explicativa ya que detallamos los procedimientos y así poder saber la causa de los fracasos que hay al momento de tratar a pacientes que presentan esta patología.

En el presente trabajo podremos encontrar temas referentes a definición, características clínicas, clasificación del Síndrome de Kelly, también de los materiales de impresión, la clasificación de los materiales de impresión, los tipos de cubeta de impresión, los pasos para la técnica de impresión, los tipos de técnicas de impresión, técnica convencional, técnica con ventana en la cubeta y por ultimo cirugía preprotésica.

\section{Materiales y métodos.}

El presente diseño de la Investigación no es experimental ya que se hizo revisión de literatura de diferentes fuentes bibliográficas, este proceso de investigación se inició con un problema. Para que este problema pueda ser investigado debíamos formularlo adecuadamente para de ahí obtener la información de documentos on-line, libros, artículos con las cuales llenaríamos nuestras expectativas y dudas para así analizar nuestras alternativas y saber cuál es la mejor.

Métodos. - en este trabajo se utilizaran los métodos teóricos: 
Se utilizó un método teórico Inductivo para el desarrollo de la investigación, tras una primera etapa de observación, análisis y clasificación de los hechos, se logra postular una hipótesis que brinda una solución al problema planteado. Se han descrito, enumerado y analizado todos los subtemas necesarios para determinar las alternativas de técnicas de impresión para mejorar la adaptación de prótesis totales en pacientes con Síndrome de Kelly.

\section{Tipo de investigación}

Documental: La información recopilada a través de documentos, artículos y libros relacionados con el Síndrome de Kelly y sus alternativas para la toma de impresión.

Descriptiva: Se refiere en la investigación cuando describimos y analizamos todo el tema en cuestión.

Correlacional: Es la relación que tienen nuestras variables

Explicativa: Porque detallará los procedimientos y así saber la causa de los fracasos en estos pacientes.

\section{Población y muestra}

No existe por ser un estudio bibliográfico, ya que es un trabajo descriptivo por lo tanto no se realizó una muestra y tampoco existe una población, no se realiza experimento alguno, sino que solo se detallará el Síndrome de Kelly y sus alternativas para la toma de impresión.

\section{Fases Metodológicas}

Este proceso tiene tres fases bien delimitadas: 
- Fase conceptual

- Fase metodológica

- Fase empírica

La fase conceptual es aquella que va desde la formación del problema de la investigación hasta cuando los objetivos del estudio hayan sido concretados.

El problema. En esta fase se realizó una descripción detallada para poder determinar las alternativas de técnicas de impresión para mejorar la adaptación de prótesis totales superiores en pacientes que presentan Síndrome de Kelly, se determinó la delimitación del problema, los objetivos que se quieren alcanzar, y la justificación de esta investigación.

Fuentes bibliográficas y trabajos de investigación. En esta fase se realizó una revisión bibliográfica de los antecedentes, bases teóricas de la investigación para el desarrollo del trabajo de titulación y poder determinar las alternativas de técnicas de impresión para mejorar la adaptación de prótesis totales superiores en pacientes que presentan Síndrome de Kelly.

Selección de la población de muestra. No existe población por ser una investigación tipo bibliográfica No experimental que incluyo revistas científicas, documentos de internet, libros y páginas web, por lo tanto no existe muestra alguna debido a que no es una investigación tipo experimental.

Instrumentos. Esta fase se la realizo a través de los buscadores virtuales como google académico, scielo, scirus, la selección de los artículos de revisión, blogs, documentos de internet, necesarios para la obtención de información para el logro total de la investigación, así como la validación por parte de expertos seleccionados para el estudio. 
La fase metodológica es en la que la idea va tomando forma. En esta fase formamos una base o estructura a la investigación que se va a desarrollar.

Elección del diseño de investigación. En esta fase se quería determinar y detallar las determinar las alternativas de técnicas de impresión para mejorar la adaptación de prótesis totales superiores en pacientes que presentan Síndrome de Kelly.

Definición de los sujetos del estudio. No existen objetos de estudio dentro del trabajo de investigación debido a que es bibliográfica No experimental.

Elección de las herramientas de recogida y análisis de los datos. En esta fase se aborda desde la perspectiva visual, ya que es clave importante para el resultado de la investigación. Durante mis años de estudio dentro de la Facultad Piloto de Odontología de la Universidad de Guayaquil, pude observar y darme cuenta que muchos desconocen las alternativas de técnicas de impresión para mejorar la adaptación de prótesis totales superiores en pacientes que presentan Síndrome de Kelly.

La fase empírica es la última fase, y aquella que nos llama más la atención.

Recolección de datos. En esta fase recogeremos los datos de forma sistemática utilizando las herramientas que hemos diseñado previamente.

Análisis de los datos. En esta fase se logró analizar las distintas variables que dieron como resultado de la investigación realizada. En la cual pudimos determinar las alternativas de técnicas de impresión para mejorar la adaptación de prótesis totales superiores en pacientes que presentan Síndrome de Kelly. 
Interpretación de los resultados. En esta fase se puede determinar los resultados obtenidos con la investigación realizada. Los cuales fueron que, tenemos 3 técnicas para pacientes que padecen este Síndrome; la técnica convencional elaborando una cubeta personalizada para cada paciente, la técnica con ventana en la cubeta que son para pacientes que presentan tejidos móviles en la parte anterior del vestíbulo de la boca y por último la cirugía preprotésica la cual consiste en realizarse todas las cirugías que sean necesarias antes de confeccionar una prótesis total superior.

Difusión de los resultados. Esta investigación y sus resultados deben llegar a todo estudiante y profesional de la odontología ya que, en cualquier momento llegara a nuestra consulta un paciente que tenga Síndrome de Kelly y tendremos que saber cómo tratarlos y que tratamiento brindarles. Los estudiantes y profesionales de odontología también deben estar capacitados para, identificar cuando un paciente tiene este Sindrome.

\section{Resultados.}

No existen resultados experimentales por ser un estudio netamente bibliográfico, en tal virtud se obtuvo de la investigación que el Síndrome de Kelly ocurre principalmente cuando estamos frente a un edentulismo total superior frente a un edentulismo parcial inferior con dientes remanentes anteriores que por lo general están extruidos, con reabsorción ósea e hiperplasia de la tuberosidad del maxilar además que este síndrome se clasifica en 3 tipos y a su vez cada tipo tiene 3 modificaciones que da como resultado 9 modificaciones, en el momento de rehabilitar a estos tipos de paciente con una prótesis total superior encontramos que tenemos 3 tipos de técnicas de impresión que podríamos hacer, la técnica convencional elaborando una cubeta personalizada para cada paciente, la técnica con ventana en la cubeta que son para pacientes que presentan tejidos móviles en la parte anterior del vestíbulo de la boca y por último la cirugía preprotesica la cual 
consiste en realizarse todas las cirugías que sean necesarias antes de confeccionar una prótesis total superior.

\section{Conclusiones.}

En base a los objetivos propuestos en la presente investigación hemos concluido:

Los pacientes con síndrome de Kelly es una patología caracterizada por varias características clínicas en personas portadoras de una prótesis total superior vs una prótesis parcial inferior con dientes remanentes anteriores.

Hay una amplia clasificación del síndrome de Kelly descubierto por Tolstunov la cual la divide en 3 y cada una tiene una modificación y en total son 9 divisiones las cuales nos ayudaran en el momento de hacer el plan de tratamiento.

La cubeta indicada para los pacientes que padecen esta patología es la cubeta individualizada ya que va a registrar los detalles anatómicos de cada paciente y así no tendremos problemas si la cubeta es grande o pequeña para nuestro paciente

Como material definitivo podemos decir que el mejor es la pasta cinquenólica ya que permite reproducir con fidelidad los detalles de la mucosa.

Un alto índice de pacientes que padecen el síndrome de kelly van a tener como característica clínica tejidos móviles en la parte anterior del vestíbulo de la boca, por dolor o por dinero los pacientes no se los remueven es por eso que utilizamos la técnica con ventana en la cubeta para mayor comodidad del paciente y del odontólogo y así obtener una buena adaptación de la prótesis total. 
En cambio muchos pacientes tendrán las condiciones económicas para poder realizarse cirugías preprotésicas las cuales también ayudaran a la adaptación de la prótesis y más aún si se pondrán implantes.

Los profesionales de la salud bucal han considerado como primera opción un tratamiento fijo implanto - soportado.

\section{Recomendaciones.}

Se recomienda:

Diagnosticar que tipo de síndrome de Kelly presenta el paciente para así darle un tratamiento personalizado.

Enseñar la técnica de impresión con ventana en la cubeta ya que es la que más se utiliza en estos pacientes.

Hacer todas las cirugías indicadas antes de rehabilitar al paciente con implantes.

Enseñar a los estudiantes que cursan su pregrado de la carrera de Odontología se los preparen para saber cómo tratar a los pacientes que presentan el Síndrome de Kelly y así no obtener fracasos.

\section{Bibliografía.}

1. Baladrón J, Peña López J. Síndrome combinado de Kelly y pseudoquiste mucoso en seno maxilar. Reconstrucción con elevación de seno, con injerto de cresta iliaca y sobredentadura. Maxillaris: Actualidad profesional e industrial del sector dental. 2012; 152(1): p. 85-98.

2. Espinosa Molina JC. Rehabilitación oral con prótesis total y prótesis parcial removible en un paciente con síndrome combinado de Kelly. Caso Clínico pevio a la obtencion del título de Odontologo. Quito: Universidad Internacional del Ecuador, Escuela de Odontología ; 2013. 
3. Tolstunov L. Management of biomechanical complication of implant-supported restoration of a patient with combination syndrome: a case report. J Oral Maxillofac Surg. 2009 ene; 67(1): p. 178-188.

4. Ozawa Deguchi J. Prostodoncia total. Primera reimpresión Ciudad Universitaria México D.F.: Universidad Nacional Autónoma de México; 1995.

5. Urban , Fischer. Prótesis Completas. 4th ed. Madrid: Elsevier-Masson; 2007.

6. Pignatta Cunha LDA, Passos Rocha E, Piza Pelizzer E. Prevalência da Síndrome de Kelly em usuarios de prótese parcial removível. Revista Gaúcha de Odontologia Porto Alegre. 2007; 55(4): p. 325-330.

7. Basker R, Davenport J, Thomason V. Tratamiento protésico en pacientes edéntulos Caracas: Amolca; 2012. 